\title{
Neuer Name, neue Krankheit?
}

U

Es gibt kein extrinsi-

sches Asthma mehr!

Nicht dass alle davon

Betroffenen plötzlich wunderbar geheilt worden wären - nein, nach dem Positionspapier „A revised nomenclature for allergy", das von einer Task Force der European Academy of Allergology and Clinical Immunology (EAACI) verfasst wurde, ist „extrinsisch" ein veralteter Ausdruck, der nicht mehr benutzt werden sollte. Das Positionspapier wurde überraschend publiziert (Allergy 2001; 56: 813-24). Das The-

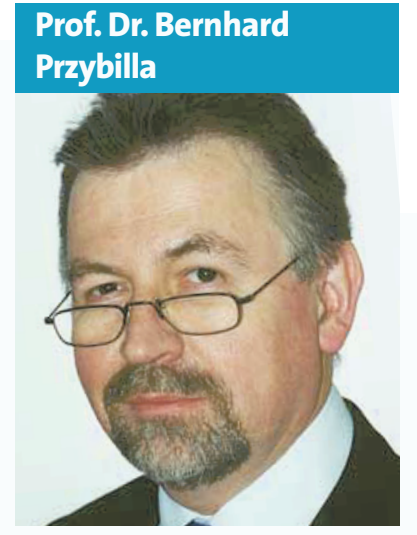

Klinik und Poliklinik für

Dermatologie und

Allergologie, Klinikum der Ludwig-MaximiliansUniversität München

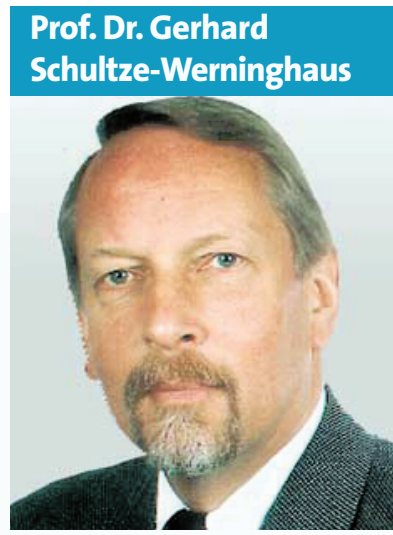

Abteilung für Pneumologie, Allergologie und Schlafmedizin, BG Kliniken Bergmannsheil Universitätsklinik, Bochum ma hätte verdient gehabt, von den Mitgliedern der EAACI breit diskutiert zu werden - eine solche Konsensfindung erfolgte leider nicht!

Die neue Nomenklatur ist einfach: Alle allergischen Erkrankungen (Rhinitis, Konjunktivitis, „Die neue Nomenklatur Asthma etc.) werist einfach: Alle aller- den eingeteilt in gischen Erkrankungen IgE-mediiert oder werden eingeteilt in IgE-assoziiert eiIgE-mediiert oder nerseits und nichtnicht-IgE-mediiert." IgE-mediiert andererseits - so wie die Weltbevölkerung in Österreicher und Nicht-Österreicher zerfällt. Bei solch eingeschränktem Gesichtsfeld geht natürlich unter, dass auch bei IgE-initiierten Erkrankungen, wie beispielsweise Asthma oder atopischem Ekzem, nichtIgE-mediierte oder -assoziierte Mechanismen eine bedeutende, oft sogar die bedeutendere Rolle spielen. Den nun per definitionem IgE-mediiert Allergiekranken ist nicht geholfen, wenn dieser für das Krankheitsmanagement wesentliche Aspekt verloren geht.

$\mathrm{Zu}$ befürchten ist auch, dass die Überbetonung von IgE als Dreh- und Angelpunkt die ohnehin häufige Überschätzung der Bedeutung von SerumIgE-Werten weiter fördert. Zwar haben IgE-Bestimmungen in der allergologischen Diagnostik einen hohen Stellen- wert, ihre Ergebnisse können jedoch für sich alleine - wie leider immer wieder angenommen - eine manifeste Allergiekrankheit weder beweisen noch widerlegen.

Auch manch anderes ist in der neuen Nomenklatur peinlich einfach dargestellt: So sind Begriffe wie Nahrungsmittelallergie oder Arzneistoffallergie ohne Bezeichnung der durch sie ausgelösten Symptome für die medizinische Versorgung des Patienten ungenügend. Die Auslösung der Arzneistoffallergie wird auf IgE-mediierte oder durch Lymphozyten vermittelte Reaktionen reduziert, ignoriert werden wichtige andere Pathomechanismen wie zytotoxische Reaktionen oder Immunkomplexreaktionen. Und Anaphylaxie als ,schwere, lebensbedrohliche, generalisierte oder systemische Überempfindlichkeitsreaktion" zu definieren ist schlicht schlampig - auch Erkrankungen wie die toxische epidermale Nekrolyse oder Vaskulitiden genügen diesen Kriterien!

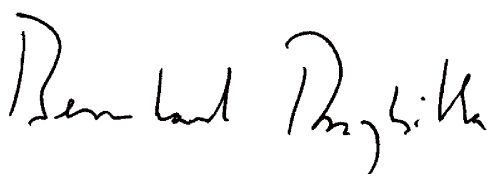

Prof. Dr. B. Przybilla
Hier ist nicht der Ort für eine umfassende Diskussion der „Revised nomenclature for allergy". Die Akzeptanz bleibt abzuwarten, aber es ist nicht anzunehmen, dass beispielsweise die Begriffe „IgEmediiertes Asthma" oder „nicht-IgE-mediiertes allergisches Asthma" den Terminus „extrinsisches Asthma“ ersetzen werden.

Die neue Nomenklatur ist also nicht der große Wurf, sie gibt aber Anlass über die Schwächen der heute gebräuchlichen, nicht selten unscharfen oder uneinheitlich verwendeten Terminologie nachzudenken. Es ist ohne Zweifel wichtig, in Wissenschaft und Patientenversorgung Begriffe präzise einzusetzen und nötigenfalls zu definieren. Das Thema „Nomenklatur" dürfte nun wieder aktuell sein und weiter bearbeitet werden, in hoffentlich nicht allzu ferner $\mathrm{Zu}$ kunft könnte die Allergologie eine einheitliche Sprache haben ....

"Die neue Nomenklatur ist nicht der große Wurf, sie gibt aber Anlass über die Schwächen der heute gebräuchlichen Terminologie nachzudenken."

Bis dahin wird zwar noch einige Zeit vergehen - dies hindert uns aber heute nicht an Informationsaustausch oder Wissensvermittlung. Die Ihnen vorliegende, von Herrn Professor Bachert als Gastherausgeber betreute Ausgabe des Allergo-Journals mit ausgewählten Themen aus der HNO-Heilkunde belegt dies im besten Sinne!

Mit kollegialen Grüßen,

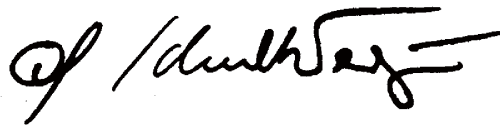

Prof. Dr. G. Schultze-Werninghaus 